\title{
Electron-impact excitation rates of Na-like ions
}

\author{
E. Kimura ${ }^{1}$, A. Ohsaki ${ }^{1}$, S. Nakazaki ${ }^{1}$, and Y. Itikawa ${ }^{2}$ \\ 1 Department of Materials Science, Faculty of Engineering, Miyazaki University, Miyazaki 889-2192, Japan \\ 2 Institute of Space and Astronautical Science, Yoshinodai, Sagamihara 229-8510, Japan
}

Received January 20; accepted March 30, 1998

\begin{abstract}
Electron excitation collision strengths for all the transitions among $3 \mathrm{~s}, 3 \mathrm{p}, 3 \mathrm{~d}, 4 \mathrm{~s}, 4 \mathrm{p}, 4 \mathrm{~d}, 4 \mathrm{f}$ states of the five Na-like ions Al III, Si IV, S VI, Ar VIII and Ca X are calculated in the R-matrix approximation. Eleven target terms are included in the expansion of the total wavefunction. These collision strengths are averaged over a Maxwellian velocity distribution to obtain effective collision strengths as a function of electron temperature. The resulting values are compared with the results of previous less elaborate calculations to find some disagreements.
\end{abstract}

Key words: atomic data

\section{Introduction}

Emission lines of Na-like ions are useful in diagnostics of astrophysical and laboratory plasmas. The relative intensities of the $3 \mathrm{p}-3 \mathrm{~s}$ and $3 \mathrm{~d}-3 \mathrm{~s}$ lines in those ions, for instance, are appropriate for the determination of temperature, because the energy separation of those levels is relatively large. (See Keenan 1993 for the general aspect of the line ratio diagnostics and Flower \& Nussbaumer 1975, Keenan et al. 1986, and Keenan 1988 for the Na-like ions.) To derive reliable values of temperature from such line ratios, we need atomic data, particularly those on the electron-impact excitation rates of ions. The present paper presents those rate coefficients for five Na-like ions (Al III, Si IV, S VI, Ar VIII, Ca X) obtained by an accurate calculation (i.e., an eleven-state R-matrix method).

In 1987, Dufton and Kingston reported the rate coefficients for Al III, Si IV and S VI. These are based on a calculation of the excitation cross section with a 5-state Rmatrix method. For Al III, Mitroy \& Norcross (1989) made a close-coupling calculation with five and nine states taken into account. They calculated also the rate coefficients. Using the data obtained by Dufton \& Kingston (1987)

Send offprint requests to: S. Nakazaki

Correspondence to: shinobu@phys.miyazaki-u.ac.jp
Table 1. Excitation energies (in Ryd) from the ground state of the Na-like ions: comparison of the present calculation and the observed values from Moore (1971) (in parentheses)

\begin{tabular}{cccccc}
\hline State & Al III & Si IV & S VI & Ar VIII & Ca X \\
\hline \multirow{3}{*}{$3 \mathrm{p}^{2} \mathrm{P}^{\circ}$} & 0.4857 & 0.6498 & 0.9698 & 1.2827 & 1.5920 \\
& $(0.4906)$ & $(0.6524)$ & $(0.9725)$ & $(1.2928)$ & $(1.6184)$ \\
$3 \mathrm{~d}^{2} \mathrm{D}$ & 1.0397 & 1.4421 & 2.2320 & 2.9956 & 3.7408 \\
& $(1.0567)$ & $(1.4615)$ & $(2.2548)$ & $(3.0315)$ & $(3.8033)$ \\
$4 \mathrm{~s}^{2} \mathrm{~S}$ & 1.1288 & 1.7431 & 3.2749 & 5.2034 & 7.5255 \\
& $(1.1497)$ & $(1.7677)$ & $(3.3077)$ & $(5.2480)$ & $(7.5894)$ \\
$4 \mathrm{p}{ }^{2} \mathrm{P}^{\circ}$ & 1.2875 & 1.9655 & 3.6253 & 5.6785 & 8.1286 \\
& $(1.3094)$ & $(1.9900)$ & $(3.6584)$ & $(5.7310)$ & $(8.2066)$ \\
$4 \mathrm{~d}^{2} \mathrm{D}$ & 1.4880 & 2.2538 & 4.0855 & 6.3086 & 8.9218 \\
& $(1.5107)$ & $(2.2783)$ & $(4.1171)$ & $(6.3562)$ & $(8.9978)$ \\
$4 \mathrm{f}^{2} \mathrm{~F}^{\circ}$ & 1.4991 & 2.2820 & 4.1696 & 6.4670 & 9.1647 \\
& $(1.5274)$ & $(2.3158)$ & $(4.2160)$ & $(6.5323)$ & $(9.2600)$ \\
$5 \mathrm{~s}^{2} \mathrm{~S}$ & 1.5293 & 2.3881 & 4.5518 & 7.2977 & 10.6205 \\
& $(1.5550)$ & $(2.4187)$ & $(4.5938)$ & $(7.4033)$ & $(10.6625)$ \\
$5 \mathrm{p}{ }^{2} \mathrm{P}^{\circ}$ & 1.6000 & 2.4895 & 4.7165 & 7.5247 & 10.9123 \\
& $(1.6262)$ & $(2.5202)$ & $(4.7584)$ & $(7.5866)$ & \\
$5 \mathrm{~d}$ & \\
& 1.6949 & 2.6253 & 4.9324 & 7.8323 & 11.2873 \\
$5 \mathrm{f}^{2} \mathrm{~F}$ & $(1.7212)$ & $(2.6563)$ & $(4.9758)$ & $(7.8833)$ & $(11.3794)$ \\
& 1.7016 & 2.6421 & 4.9801 & 7.9084 & 11.4174 \\
& $(1.7303)$ & $(2.6766)$ & $(5.0288)$ & $(7.9759)$ & $(11.5125)$ \\
\hline
\end{tabular}

and more recent R-matrix type calculations for Ti XII, Fe XVI and Ni XVIII, Keenan et al. (1996) estimated rate coefficients for a number of Na-like ions, with an interpolation and scaling procedure. Keenan et al. (1996) explicitly reported the rate coefficients for the Na-like ions with $Z=15,17-21,23-25$ and 27.

Recently, Nakazaki et al. (1996, referred to as Paper I) made an elaborate calculation (i.e., with a seven and eleven state R-matrix method) for Ar VIII. They showed quantitatively the importance of resonant collisions and some details of the relevant differential cross section. 
The present paper extends their calculation to four other Na-like ions (Al III, Si IV, S VI and Ca X). From the collision cross sections obtained, the rate coefficients are calculated for all the transitions among $3 \mathrm{~s}, 3 \mathrm{p}, 3 \mathrm{~d}$, $4 \mathrm{~s}, 4 \mathrm{p}, 4 \mathrm{~d}, 4 \mathrm{f}$ states for the four Na-like ions and Ar VIII. The resulting values are expected to be more accurate and more comprehensive than the previous ones cited above and will serve as a standard for future applications.

\section{Theory}

\subsection{Target wavefunctions}

The lowest eleven states of the target ion, i.e., $3 \mathrm{~s}, 3 \mathrm{p}$, $3 \mathrm{~d}, 4 \mathrm{~s}, 4 \mathrm{p}, 4 \mathrm{~d}, 4 \mathrm{f}, 5 \mathrm{~s}, 5 \mathrm{p}, 5 \mathrm{~d}$ and $5 \mathrm{f}$ states, are included in the present calculation. Each state is represented by a configuration interaction wavefunction. The radial part of the one-electron orbitals is expressed in the Slater form

$P_{n l}(r)=\sum_{j=1}^{K} C_{j} r^{p_{j}} \exp \left(-\xi_{j} r\right)$.

The coefficients $C_{j}$ and the parameters $p_{j}$ and $\xi_{j}$ for the $1 \mathrm{~s}, 2 \mathrm{~s}, 2 \mathrm{p}$ and $3 \mathrm{~s}$ orbitals are taken from the paper by Clementi \& Roetti (1974), while those for the other orbitals are obtained with the computer code of Hibbert (1975) by optimizing those orbitals on the energy of the corresponding states.

Excitation energies obtained in the present calculation are compared in Table 1 with the experimental results (Moore 1971). The excitation energies of Ar VIII are different from those of the Paper I owing to the use of $3 \mathrm{~d}$ orbital with three terms. Oscillator strengths for the optically allowed transitions from the ground state to $3 \mathrm{p}$ and $4 \mathrm{p}$ states are shown in Table 2. Both the excitation energies and the oscillator strengths obtained in the present calculation are in good agreement with those of experiment and those of other calculations. This indicates the reliability of the present wavefunction of the target ions.

\subsection{Collision calculations}

The R-matrix theory of electron-ion collisions has been described in detail by Burke \& Robb (1975). The total wavefunction representing the electron-ion collision system is expanded in a sphere with radius $r_{\mathrm{a}}$ as follows:

$$
\begin{array}{r}
\Psi_{k}=\mathcal{A} \sum_{i j} c_{i j k} \Phi_{i}(1, \cdots, \\
\left.+\hat{\mathbf{r}}_{N+1}, \sigma_{N+1}\right) u_{i j}\left(r_{N+1}\right) \\
+\sum_{j} d_{j k} \phi_{j}(1, \cdots, N+1),
\end{array}
$$

where $\mathcal{A}$ is the anti-symmetrization operator, $\Phi_{i}$ the channel function representing the target state coupled with the spin and angular functions for the scattering electron, $u_{i j}$ the continuum basis orbitals for the scattered electron, and $\phi_{j}$ twelve-electron bound configurations formed from fourteen bound orbitals. Details of the basis functions $u_{i j}$ and $\phi_{j}$ are described in Paper I. The coefficients $c_{i j k}$ and $d_{j k}$ are determined by diagonalizing the total Hamiltonian of the whole system with the basis set expansion defined by Eq. (2).

We use the computer code of Berrington et al. (1978) to calculate the R-matrix on the boundary of the sphere, whose radius $\left(r_{\mathrm{a}}\right)$ is taken to be $27.8,21.0,14.2,11.0$ and 8.8 a.u. for $\mathrm{Al}$ III, Si IV, S VI, Ar VIII and Ca X, respectively. We include 30 continuum orbitals for each ion. In the outer region of the sphere, a set of close-coupling equation is solved for the partial waves $L=0-10$, using the asymptotic code STGF of Berrington et al. (1987). Contributions from the partial waves higher than those are evaluated by using a two-state close-coupling approximation of Henry et al. (1981) and the Coulomb-Born approximation of Takagishi et al. (1995).

\subsection{Calculation of rate coefficients}

Excitation rate coefficients $C$ (in $\left.\mathrm{cm}^{3} \mathrm{~s}^{-1}\right)$ for a transition $i$ to $f$ is calculated using the following formula,

$C(i \rightarrow f)=\frac{8.62910^{-6}}{g_{i} \sqrt{T_{\mathrm{e}}}} \exp \left(-\Delta E_{i f} / k T_{\mathrm{e}}\right) \gamma(i \rightarrow f)$

where $\gamma$ is the effective collision strength defined by

$\gamma(i \rightarrow f)=\int_{0}^{\infty} \Omega_{i \rightarrow f} \exp \left(-\varepsilon_{f} / k T_{\mathrm{e}}\right) \mathrm{d}\left(\varepsilon_{f} / k T_{\mathrm{e}}\right)$

Here $\varepsilon_{f}$ is the energy of the electron after the collision, $g_{i}$ the statistical weight of the level $i, \Delta E_{i f}$ the excitation energy, and $\Omega_{i \rightarrow f}$ the collision strength. The temperature $T_{\mathrm{e}}$ is expressed in $\mathrm{K}$. To obtain the rate coefficient at high temperature, we fit the present collision strengths in the region where no resonances appear to an analytic form

$\Omega_{i \rightarrow f}=A+B / X+C / X^{2}+D / X^{3}+E \ln X$,

where $X=k_{i}^{2} / \Delta E_{i f}$, where $k_{i}^{2}$ is the incident energy in Ryd and $\Delta E_{i f}$ is given in Ryd. For a dipole-allowed transition, the form is used with $D=0$ and $E=4 g_{i} f_{i f} / \Delta E_{i f}$, where $f_{\text {if }}$ is the oscillator strength for $i \rightarrow f$. The form (5) with $E=0$ is used for optically forbidden cases. Thus the effective collision strength is evaluated with the collision strengths calculated in the resonance region and with the Eq. (5) otherwise.

\section{Results and discussion}

Effective collision strengths obtained for all the transitions among $3 \mathrm{~s}, 3 \mathrm{p}, 3 \mathrm{~d}, 4 \mathrm{~s}, 4 \mathrm{p}, 4 \mathrm{~d}$ and $4 \mathrm{f}$ states are given in Tables 3-7 for Al III, Si IV, S VI, Ar VIII and $\mathrm{Ca} \mathrm{X}$, respectively. The temperature range considered is around the temperature $\left(T_{\max }\right)$ of maximum fractional abundance in ionization equilibrium of the respective 
Table 2. Oscillator strengths

\begin{tabular}{l|ccc|ccc}
\hline Ion & \multicolumn{3}{|c|}{$3 \mathrm{~s}-3 \mathrm{p}$} & \multicolumn{3}{c}{$3 \mathrm{~s}-4 \mathrm{p}$} \\
\hline & \multicolumn{2}{|c}{ Present } & Wiese et al. (1969) & \multicolumn{2}{c}{ Present } & Wiese et al. (1969) \\
& $l$ & $v$ & & $l$ & $v$ & \\
\hline Al III & 0.8889 & 0.8442 & 0.875 & 0.0129 & 0.0125 & 0.011 \\
Si IV & 0.8154 & 0.7721 & 0.803 & 0.0291 & 0.0305 & 0.033 \\
S VI & 0.6885 & 0.6557 & 0.636 & 0.0735 & 0.0739 & 0.071 \\
Ar VIII & 0.5914 & 0.5624 & 0.57 & 0.1189 & 0.1161 & 0.12 \\
Ca X & 0.5179 & 0.4944 & 0.525 & 0.1542 & 0.1508 & 0.145 \\
\hline
\end{tabular}

Table 3. Effective collision strengths for Al III

\begin{tabular}{|c|c|c|c|c|c|c|c|c|c|c|c|}
\hline Transition & 3.6 & 3.8 & 4.0 & 4.2 & 4.4 & $\begin{array}{c}\log _{10} T_{\mathrm{e}} \\
4.6\end{array}$ & 4.8 & 5.0 & 5.2 & 5.4 & 5.6 \\
\hline \multicolumn{12}{|l|}{$3 \mathrm{~s}-$} \\
\hline $3 p$ & $1.49+1$ & $.54+1$ & $1.60+1$ & $1.67+1$ & $1.74+1$ & $1.83+1$ & $1.94+1$ & $2.10+1$ & $2.34+1$ & $2.69+1$ & $3.13+1$ \\
\hline $3 d$ & $2.65+0$ & $2.80+0$ & $2.89+0$ & $2.93+0$ & $.93+0$ & $2.92+0$ & $2.95+0$ & $3.04+0$ & $3.21+0$ & $3.44+0$ & $.70+0$ \\
\hline $4 \mathrm{~s}$ & $1.01+0$ & $1.03+0$ & $1.02+0$ & $1.00+0$ & $9.66-1$ & $9.30-1$ & $9.08-1$ & $9.07-1$ & $9.28-1$ & $9.64-1$ & $1.01+0$ \\
\hline $4 p$ & $7.81-1$ & $7.74-1$ & $7.63-1$ & $7.36-1$ & $6.91-1$ & $6.40-1$ & $5.95-1$ & $5.63-1$ & $5.43-1$ & $5.32-1$ & $5.26-1$ \\
\hline $4 \mathrm{~d}$ & $6.09-1$ & $5.60-1$ & $5.19-1$ & $4.84-1$ & $4.57-1$ & $4.38-1$ & $4.28-1$ & $4.24-1$ & $4.25-1$ & $4.27-1$ & $4.31-1$ \\
\hline $4 \mathrm{f}$ & $4.63-1$ & $4.53-1$ & $4.43-1$ & $4.34-1$ & $4.29-1$ & $4.28-1$ & $4.32-1$ & $4.39-1$ & $4.46-1$ & $4.48-1$ & $4.45-1$ \\
\hline \multicolumn{12}{|c|}{ (2) } \\
\hline $3 d$ & $2.66+1$ & $2.89+1$ & $3.07+1$ & $3.22+1$ & $3.37+1$ & $3.54+1$ & $3.81+1$ & $4.23+1$ & $4.86+1$ & $5.74+1$ & $6.88+1$ \\
\hline $4 \mathrm{~s}$ & $2.98+0$ & $3.01+0$ & $2.99+0$ & $2.94+0$ & $2.85+0$ & $2.78+0$ & $2.80+0$ & $2.97+0$ & 3.3 & 4.0 & $5.06+0$ \\
\hline $4 p$ & $6.13+0$ & $6.10+0$ & $6.06+0$ & $5.92+0$ & $5.67+0$ & $5.41+0$ & $5.22+0$ & $5.15+0$ & 5.20 & $5.36+0$ & $5.60+0$ \\
\hline $4 d$ & $3.79+0$ & $3.56+0$ & $3.36+0$ & $3.18+0$ & $3.03+0$ & $2.94+0$ & $2.91+0$ & $2.93+0$ & $2.97+0$ & $3.05+0$ & $3.12+0$ \\
\hline $4 \mathrm{f}$ & $3.80+0$ & $3.81+0$ & $3.92+0$ & $4.13+0$ & $4.21+0$ & $4.11+0$ & $4.05+0$ & $4.13+0$ & $4.33+0$ & $4.59+0$ & $4.86+0$ \\
\hline \multicolumn{12}{|c|}{$\begin{array}{lll} & 0.00 \\
\end{array}$} \\
\hline $4 \mathrm{~s}$ & $9.36+0$ & $1.03+1$ & $1.07+1$ & $1.07+1$ & $1.03+1$ & $9.71+0$ & $8.90+0$ & $8.07+0$ & $7.58+0$ & $7.48+0$ & $7.61+0$ \\
\hline $4 p$ & $4.29+1$ & $4.16+1$ & $4.07+1$ & $4.02+1$ & $4.07+1$ & $4.07+1$ & $3.91+1$ & $3.84+1$ & $4.13+1$ & $4.80+1$ & $5.75+1$ \\
\hline $4 \mathrm{~d}$ & $3.28+1$ & $2.93+1$ & $2.65+1$ & $2.45+1$ & $2.35+1$ & $2.30+1$ & $2.18+1$ & $2.05+1$ & $1.98+1$ & $2.00+1$ & $2.05+1$ \\
\hline \multirow{2}{*}{\multicolumn{12}{|c|}{ 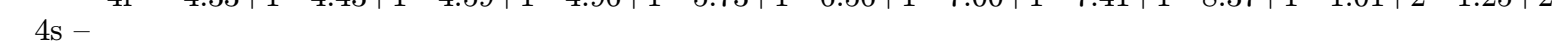 }} \\
\hline & & & & & & & & & & & \\
\hline $4 p$ & $4.62+1$ & $4.81+1$ & $5.10+1$ & $5.66+1$ & $6.71+1$ & $7.70+1$ & $8.13+1$ & $8.57+1$ & $9.79+1$ & $1.19+2$ & $1.47+2$ \\
\hline $4 d$ & $1.13+1$ & $1.12+1$ & $1.25+1$ & $1.50+1$ & $1.64+1$ & $1.57+1$ & $1.38+1$ & $1.25+1$ & $1.23+1$ & $1.28+1$ & $1.35+1$ \\
\hline $4 \mathrm{f}$ & $7.91+0$ & $8.16+0$ & $9.40+0$ & $1.12+1$ & $1.20+1$ & $1.12+1$ & $9.57+0$ & $8.24+0$ & $7.47+0$ & $7.04+0$ & $6.73+0$ \\
\hline \multicolumn{12}{|c|}{ 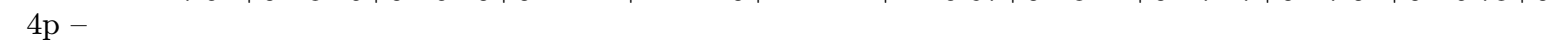 } \\
\hline $4 d$ & & & & & & & & & & & \\
\hline \multicolumn{12}{|l|}{$4 d-$} \\
\hline $4 \mathrm{f}$ & $6.58+2$ & $7.42+2$ & $9.97+2$ & $1.36+3$ & $1.57+3$ & $1.51+3$ & $1.29+3$ & $1.08+3$ & $9.72+2$ & $9.49+2$ & $9.67+2$ \\
\hline
\end{tabular}

species. According to the calculation by Arnaud \& Rothenflug (1985), Log $T_{\max }$ for Al III, Si IV, S VI, Ar VIII and Ca X are, respectively, 4.6, 4.8, 5.3, 5.6 and 5.8.

For Al III, Si IV and S VI, Dufton \& Kingston (1987) reported their rate coefficients for the transitions among $3 \mathrm{~s}, 3 \mathrm{p}, 3 \mathrm{~d}, 4 \mathrm{~s}$ and $4 \mathrm{p}$. They took account of only 5 -states $(3 \mathrm{~s}, 3 \mathrm{p}, 3 \mathrm{~d}, 4 \mathrm{~s}$ and $4 \mathrm{p})$ in their calculation. In principle, the present 11-state calculation should be much better than theirs, especially for the $n=3-4$ transition. Figure 1 shows a typical example (the $3 \mathrm{~s}-4 \mathrm{p}$ ) of the comparison between their result and the present one. There is a discrepancy of up to a factor of two. Mitroy \& Norcross (1989) also obtained the rate coefficient for Al III. They used five atomic states and four pseudostates $(\overline{4 \mathrm{~d}}, \overline{4 \mathrm{f}}, \overline{5 \mathrm{~s}}$ and $\overline{5 \mathrm{p}}$ ) in the close-coupling approximation. Their results are much smaller than those of present calculation for the $3 \mathrm{~s}-4 \mathrm{p}$ transition, whereas for the $n=3-3$ transitions the agreement between the two calculations is good within
$10 \%$. It is seen that the effect of including $n=5$ states in the present calculation is important. Keenan et al. (1996) determined the rate coefficients for Na-like ions, based on the interpolation of results in the R-matrix calculations available for some particular Na-like species. When compared with the present calculation, their data in some cases show a large disagreement. Figure 2 shows one example. The procedure taken by Keenan et al. (1996) implicitly assumes a scaling of the effective collision strengths along the isoelectronic sequence.

There is no experimental determination of the rate coefficients for the Na-like ions considered here. Experimental data are available, however, for the excitation cross sections of Si IV (Wåhlin et al. 1991) and Ar VIII (Guo et al. 1993). In Fig. 3, we compare the present cross section for the $3 \mathrm{~s}-3 \mathrm{p}$ excitation of Si IV with the corresponding experimental values. No adjustment has been done of the absolute magnitude in both the cross 
Table 4. Effective collision strengths for Si IV

\begin{tabular}{|c|c|c|c|c|c|c|c|c|c|c|c|}
\hline Transition & 3.8 & 4.0 & 4.2 & 4.4 & 4.6 & $\begin{array}{c}\log _{10} T_{\mathrm{e}} \\
4.8\end{array}$ & 5.0 & 5.2 & 5.4 & 5.6 & 5.8 \\
\hline \multicolumn{12}{|l|}{$3 \mathrm{~s}-$} \\
\hline $3 p$ & $1.50+1$ & $1.50+1$ & $.51+1$ & $1.53+1$ & $1.58+1$ & $1.63+1$ & $1.71+1$ & $1.82+1$ & $1.99+1$ & $2.22+1$ & $2.53+1$ \\
\hline $3 \mathrm{~d}$ & $2.33+0$ & $2.43+0$ & $2.50+0$ & $2.54+0$ & $2.53+0$ & $2.50+0$ & $2.48+0$ & $2.50+0$ & $2.57+0$ & $2.68+0$ & $2.82+0$ \\
\hline $4 \mathrm{~s}$ & $6.93-1$ & $7.43-1$ & $7.66-1$ & $7.65-1$ & $7.48-1$ & $7.26-1$ & $7.09-1$ & $7.03-1$ & $7.08-1$ & $7.22-1$ & $7.43-1$ \\
\hline $4 p$ & $5.67-1$ & $5.62-1$ & $5.52-1$ & $5.28-1$ & $4.88-1$ & $4.42-1$ & $3.99-1$ & $3.64-1$ & $3.38-1$ & $3.20-1$ & $3.10-1$ \\
\hline $4 d$ & $3.81-1$ & $3.64-1$ & $3.45-1$ & $3.23-1$ & $2.99-1$ & $2.77-1$ & $2.57-1$ & $2.41-1$ & $2.27-1$ & $2.16-1$ & $2.08-1$ \\
\hline $4 \mathrm{f}$ & $3.72-1$ & $3.69-1$ & $3.64-1$ & $3.56-1$ & $3.49-1$ & $3.45-1$ & $3.45-1$ & $3.49-1$ & $3.54-1$ & $3.58-1$ & $3.61-1$ \\
\hline \multicolumn{12}{|l|}{$3 p-$} \\
\hline $3 \mathrm{~d}$ & $2.56+1$ & 2.66 & 2.76 & 2.86 & $2.9 ?$ & $3.10+1$ & $3.26+1$ & 3.50 & $3.8 \mathrm{~s}$ & $4.43+1$ & $5.12+1$ \\
\hline $4 \mathrm{~s}$ & $1.87+0$ & $2.03+0$ & $2.07+0$ & $2.01+0$ & $1.91+0$ & $1.79+0$ & $1.72+0$ & $1.74+0$ & $1.90+0$ & $2.21+0$ & $2.67+0$ \\
\hline $4 p$ & $4.36+0$ & $4.45+0$ & $4.46+0$ & $4.38+0$ & $4.22+0$ & $3.97+0$ & $3.75+0$ & $3.61+0$ & $3.58+0$ & $3.64+0$ & $3.76+0$ \\
\hline $4 d$ & $2.42+0$ & $2.30+0$ & $2.21+0$ & $2.14+0$ & $2.00+0$ & $1.82+0$ & $1.65+0$ & $1.53+0$ & $1.44+0$ & $1.38+0$ & $1.32+0$ \\
\hline $4 \mathrm{f}$ & $2.67+0$ & $2.73+0$ & $2.87+0$ & $3.00+0$ & $2.99+0$ & $2.90+0$ & $2.86+0$ & $2.92+0$ & $3.06+0$ & $3.25+0$ & $3.48+0$ \\
\hline $4 \mathrm{~s}$ & 5 & 8 & 31 & 0 & 6.0 & $5.52+0$ & $4.84+0$ & & 3.8 & & $3.52+0$ \\
\hline $4 p$ & $1.99+1$ & $2.07+1$ & $2.09+1$ & $2.06+1$ & $1.99+1$ & $1.86+1$ & $1.69+1$ & $1.58+1$ & $1.61+1$ & $1.78+1$ & $2.05+1$ \\
\hline $4 d$ & $1.90+1$ & $1.76+1$ & $1.72+1$ & $1.78+1$ & $1.78+1$ & $1.64+1$ & $1.43+1$ & $1.27+1$ & $1.19+1$ & $1.17+1$ & $1.17+1$ \\
\hline $4 \mathrm{f}$ & $2.86+1$ & $3.04+1$ & $3.54+1$ & $4.28+1$ & $4.65+1$ & $4.46+1$ & $4.12+1$ & $4.10+1$ & $4.53+1$ & $5.33+1$ & $6.39+1$ \\
\hline \multicolumn{12}{|c|}{$2.00 \mid 10.04 / 1 \quad 0.04 / 1 \quad 7.2011$} \\
\hline $4 p$ & $4.61+1$ & $4.76+1$ & $4.99+1$ & $5.54+1$ & $6.48+1$ & $7.20+1$ & $7.35+1$ & $7.50+1$ & $8.23+1$ & $9.60+1$ & $1.14+2$ \\
\hline $4 d$ & $1.10+1$ & $1.09+1$ & $1.16+1$ & $1.34+1$ & $1.46+1$ & $1.40+1$ & $1.25+1$ & $1.13+1$ & $1.09+1$ & $1.10+1$ & $1.14+1$ \\
\hline $4 \mathrm{f}$ & $6.33+0$ & $6.35+0$ & $6.87+0$ & $7.78+0$ & $8.16+0$ & $7.56+0$ & $6.48+0$ & $5.55+0$ & $4.96+0$ & $4.60+0$ & $4.34+0$ \\
\hline \multicolumn{12}{|l|}{$4 p-$} \\
\hline $4 \mathrm{~d}$ & $8.61+1$ & $8.89+1$ & $1.05+2$ & 1.3 & $1.60+2$ & $1.60+2$ & 1.46 & $1.38+2$ & & & \\
\hline \multicolumn{12}{|c|}{$\begin{array}{llll}4.29+1 & 4.07+1 & 3.52+1 & 3.07+1\end{array}$} \\
\hline $4 \mathrm{f}$ & $3.89+2$ & $4.28+2$ & $5.48+2$ & $7.35+2$ & $8.56+2$ & $8.36+2$ & $7.25+2$ & $6.19+2$ & $5.69+2$ & $5.66+2$ & $5.90+2$ \\
\hline
\end{tabular}

Table 5. Effective collision strengths for S VI

\begin{tabular}{|c|c|c|c|c|c|c|c|c|c|c|c|}
\hline Transition & 4.3 & 4.5 & 4.7 & 4.9 & 5.1 & $\begin{array}{c}\log _{10} T_{\mathrm{e}} \\
5.3\end{array}$ & 5.5 & 5.7 & 5.9 & 6.1 & 6.3 \\
\hline \multicolumn{12}{|l|}{$3 \mathrm{~s}-$} \\
\hline $3 p$ & $1.14+1$ & $1.15+1$ & $1.17+1$ & $1.20+1$ & $1.23+1$ & $1.27+1$ & $1.34+1$ & $1.43+1$ & $1.56+1$ & $1.73+1$ & $1.91+1$ \\
\hline $3 \mathrm{~d}$ & $1.48+0$ & $1.54+0$ & $1.59+0$ & $1.62+0$ & $1.62+0$ & $1.61+0$ & $1.61+0$ & $1.62+0$ & $1.66+0$ & $1.72+0$ & $1.77+0$ \\
\hline $4 \mathrm{~s}$ & $5.32-1$ & $5.31-1$ & $5.15-1$ & $4.92-1$ & $4.70-1$ & $4.53-1$ & $4.43-1$ & $4.40-1$ & $4.44-1$ & $4.52-1$ & $4.62-1$ \\
\hline $4 p$ & $3.10-1$ & $3.02-1$ & $2.82-1$ & $2.53-1$ & $2.21-1$ & $1.93-1$ & $1.72-1$ & $1.58-1$ & $1.53-1$ & $1.56-1$ & $1.69-1$ \\
\hline $4 d$ & $2.08-1$ & $1.97-1$ & $1.82-1$ & $1.64-1$ & $1.45-1$ & $1.29-1$ & $1.15-1$ & $1.04-1$ & $9.67-2$ & $9.27-2$ & $9.16-2$ \\
\hline $4 \mathrm{f}$ & $2.76-1$ & $2.78-1$ & $2.74-1$ & $2.69-1$ & $2.64-1$ & $2.61-1$ & $2.60-1$ & $2.62-1$ & $2.66-1$ & $2.70-1$ & $2.73-1$ \\
\hline \multicolumn{12}{|l|}{$3 p-$} \\
\hline $3 \mathrm{~d}$ & $1.84+1$ & $92+1$ & $2.01+1$ & $2.09+1$ & $.18+1$ & $2.28+1$ & $2.41+1$ & $2.60+1$ & $2.84+1$ & $3.13+1$ & $3.46+1$ \\
\hline $4 \mathrm{~s}$ & $1.22+0$ & $1.21+0$ & 1.1 & 9.9 & 8.7 & $7.92-1$ & 7.5 & 7.8 & 8. & 1.0 & $1.27+0$ \\
\hline $4 p$ & $2.67+0$ & $2.61+0$ & $2.52+0$ & $2.40+0$ & $2.27+0$ & $2.15+0$ & $2.07+0$ & $2.05+0$ & $2.07+0$ & $2.11+0$ & $2.17+0$ \\
\hline $4 d$ & $1.17+0$ & $1.13+0$ & $1.13+0$ & $1.10+0$ & $9.92-1$ & $8.46-1$ & $7.20-1$ & $6.37-1$ & $5.96-1$ & $5.91-1$ & $6.21-1$ \\
\hline $4 \mathrm{f}$ & $1.70+0$ & $1.77+0$ & $1.84+0$ & $1.85+0$ & $1.82+0$ & $1.80+0$ & $1.82+0$ & $1.89+0$ & $2.00+0$ & $2.15+0$ & $2.31+0$ \\
\hline $3 d-4 s$ & & & & & & & & & & & $07+0$ \\
\hline $4 \mathrm{p}$ & & 9.2 & & & & $5.29+0$ & & & & & $59+0$ \\
\hline $4 d$ & & & & & & $6.91+0$ & & & & & $5+0$ \\
\hline $4 \mathrm{f}$ & $1.56+1$ & $1.75+1$ & $2.04+1$ & $2.20+1$ & $2.14+1$ & $2.03+1$ & $2.03+1$ & $2.18+1$ & $2.46+1$ & $2.85+1$ & $3.33+1$ \\
\hline \multicolumn{12}{|c|}{ 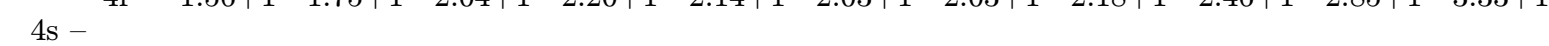 } \\
\hline $4 p$ & $4.13+1$ & $4.27+1$ & $4.65+1$ & $5.29+1$ & $5.71+1$ & $5.69+1$ & $5.66+1$ & $5.95+1$ & $6.58+1$ & $7.42+1$ & $8.36+1$ \\
\hline $4 \mathrm{~d}$ & $7.31+0$ & $7.80+0$ & $8.97+0$ & $9.90+0$ & $9.72+0$ & $8.78+0$ & $7.94+0$ & $7.53+0$ & $7.45+0$ & $7.53+0$ & $7.66+0$ \\
\hline $4 \mathrm{f}$ & $3.07+0$ & $3.31+0$ & $3.70+0$ & $3.87+0$ & $3.60+0$ & $3.11+0$ & $2.67+0$ & $2.37+0$ & $2.19+0$ & $2.06+0$ & $1.96+0$ \\
\hline \multicolumn{12}{|c|}{ (2. } \\
\hline $4 \mathrm{f}$ & $1.52+1$ & $1.72+1$ & $\begin{array}{l}1.04+2 \\
2.05+1\end{array}$ & $2.25+1$ & $2.15+1$ & $1.88+1$ & $1.65+1$ & $1.52+1$ & $1.48+1$ & $\begin{array}{l}1.42+2 \\
1.47+1\end{array}$ & $\begin{array}{l}1.00+2 \\
1.46+1\end{array}$ \\
\hline 4f & $2.01+2$ & $2.42+2$ & $3.10+2$ & $3.58+2$ & $3.51+2$ & $3.10+2$ & $2.74+2$ & $2.62+2$ & $2.72+2$ & $2.92+2$ & $3.16+2$ \\
\hline
\end{tabular}


Table 6. Effective collision strengths for Ar VIII

\begin{tabular}{|c|c|c|c|c|c|c|c|c|c|c|c|}
\hline Transition & 4.6 & 4.8 & 5.0 & 5.2 & 5.4 & $\begin{array}{c}\log _{10} T_{\mathrm{e}} \\
5.6\end{array}$ & 5.8 & 6.0 & 6.2 & 6.4 & 6.6 \\
\hline \multicolumn{12}{|l|}{$3 \mathrm{~s}-$} \\
\hline $3 p$ & $8.67+0$ & $8.84+0$ & $9.01+0$ & $9.19+0$ & $9.41+0$ & $9.74+0$ & $1.03+1$ & $1.10+1$ & $1.19+1$ & $1.30+1$ & $1.41+1$ \\
\hline $3 \mathrm{~d}$ & $1.22+0$ & $1.20+0$ & $1.19+0$ & $1.18+0$ & $1.16+0$ & $1.13+0$ & $1.12+0$ & $1.11+0$ & $1.12+0$ & $1.14+0$ & $1.17+0$ \\
\hline $4 \mathrm{~s}$ & $3.53-1$ & $3.46-1$ & $3.34-1$ & $3.20-1$ & $3.08-1$ & $3.00-1$ & $2.97-1$ & $2.97-1$ & $3.01-1$ & $3.07-1$ & $3.14-1$ \\
\hline $4 p$ & $2.15-1$ & $1.97-1$ & $1.75-1$ & $1.51-1$ & $1.29-1$ & $1.13-1$ & $1.03-1$ & $9.94-2$ & $1.04-1$ & $1.18-1$ & $1.41-1$ \\
\hline $4 d$ & $1.50-1$ & $1.39-1$ & $1.26-1$ & $1.12-1$ & $9.93-2$ & $8.91-2$ & $8.20-2$ & $7.83-2$ & $7.79-2$ & $8.03-2$ & $8.47-2$ \\
\hline $4 \mathrm{f}$ & $2.35-1$ & $2.29-1$ & $2.23-1$ & $216-1$ & $2.11-1$ & $2.07-1$ & $2.06-1$ & $2.07-1$ & $2.08-1$ & $2.10-1$ & $2.12-1$ \\
\hline \multicolumn{12}{|l|}{$3 p-$} \\
\hline $3 d$ & $1.44+1$ & 1.47 & 1.5 & 1.56 & 1.61 & $1.67+1$ & 1.7 & $1.82+1$ & 1.93 & $2.06+1$ & $2.21+1$ \\
\hline $4 \mathrm{~s}$ & $9.44-1$ & $8.67-1$ & $7.50-1$ & $6.27-1$ & $5.23-1$ & $4.51-1$ & $4.17-1$ & $4.20-1$ & $4.59-1$ & $5.32-1$ & $6.37-1$ \\
\hline $4 p$ & $1.80+0$ & $1.74+0$ & $1.66+0$ & $1.57+0$ & $1.47+0$ & $1.40+0$ & $1.36+0$ & $1.35+0$ & $1.36+0$ & $1.38+0$ & $1.41+0$ \\
\hline $4 d$ & $8.79-1$ & $7.94-1$ & $7.09-1$ & $6.25-1$ & $5.50-1$ & $4.92-1$ & $4.58-1$ & $4.52-1$ & $4.76-1$ & $5.35-1$ & $6.30-1$ \\
\hline $4 \mathrm{f}$ & $1.27+0$ & $1.26+0$ & $1.24+0$ & $1.20+0$ & $1.18+0$ & $1.19+0$ & $1.24+0$ & $1.31+0$ & $1.40+0$ & $1.51+0$ & $1.62+0$ \\
\hline \multicolumn{12}{|l|}{$3 \mathrm{~d}$} \\
\hline $4 \mathrm{~s}$ & $1+0$ & $1.77+0$ & & & +0 & $7.99-1$ & 6.40 & 0 & & & \\
\hline $4 p$ & $5.88+0$ & $5.08+0$ & $4.26+0$ & $3.48+0$ & $2.80+0$ & $2.27+0$ & $1.95+0$ & $1.80+0$ & $1.80+0$ & $1.92+0$ & $2.13+0$ \\
\hline $4 d$ & $6.08+0$ & $5.61+0$ & $5.26+0$ & $4.84+0$ & $4.31+0$ & $3.85+0$ & $3.53+0$ & $3.32+0$ & $3.21+0$ & $3.14+0$ & $3.12+0$ \\
\hline $4 \mathrm{f}$ & $1.03+1$ & $1.06+1$ & $1.10+1$ & $1.08+1$ & $1.03+1$ & $1.03+1$ & $1.09+1$ & $1.21+1$ & $1.39+1$ & $1.62+1$ & $1.89+1$ \\
\hline \multicolumn{12}{|c|}{ 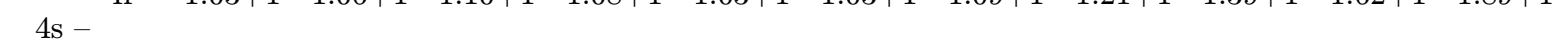 } \\
\hline $4 p$ & $3.24+1$ & $3.37+1$ & $3.72+1$ & $4.14+1$ & $4.27+1$ & $4.18+1$ & $4.19+1$ & $4.40+1$ & 4.7 & $5.27+1$ & $5.80+1$ \\
\hline $4 d$ & $4.95+0$ & $5.32+0$ & $5.96+0$ & $6.26+0$ & +0 & $5.38+0$ & +0 & +0 & +0 & $4.96+0$ & $5.03+0$ \\
\hline $4 \mathrm{f}$ & $1.79+0$ & $1.89+0$ & $2.02+0$ & $2.01+0$ & $1.81+0$ & $1.56+0$ & $1.37+0$ & $1.25+0$ & $1.17+0$ & $1.12+0$ & $1.09+0$ \\
\hline \multicolumn{12}{|l|}{$4 p-$} \\
\hline $4 d$ & $5.88+1$ & $6.65+1$ & $7.95+1$ & $8.75+1$ & $8.51+1$ & $7.89+1$ & 7.7 & 8.1 & $9.02+1$ & $1.01+2$ & $1.13+2$ \\
\hline $4 \mathrm{f}$ & \multicolumn{10}{|c|}{ 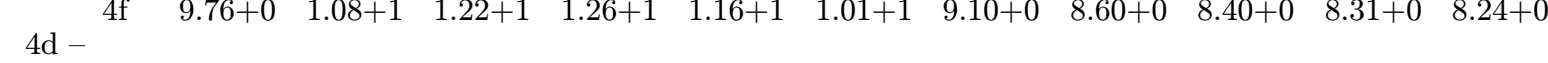 } & $8.24+0$ \\
\hline $4 \mathrm{f}$ & $1.26+2$ & $1.52+2$ & $1.89+2$ & $2.07+2$ & $1.96+2$ & $1.73+2$ & $1.59+2$ & $1.55+2$ & $1.60+2$ & $1.68+2$ & $1.78+2$ \\
\hline
\end{tabular}

Table 7. Effective collision strengths for Ca X

\begin{tabular}{|c|c|c|c|c|c|c|c|c|c|c|c|}
\hline Transition & 4.8 & 5.0 & 5.2 & 5.4 & 5.6 & $\begin{array}{c}\log _{10} T_{\mathrm{e}} \\
5.8\end{array}$ & 6.0 & 6.2 & 6.4 & 6.6 & 6.8 \\
\hline \multicolumn{12}{|l|}{$3 \mathrm{~s}-$} \\
\hline $3 p$ & $6.88+0$ & $6.96+0$ & $7.05+0$ & $.15+0$ & $.30+0$ & $7.56+0$ & $7.94+0$ & $3.46+0$ & $9.06+0$ & $9.71+0$ & $1.04+1$ \\
\hline $3 d$ & $9.51-1$ & $9.09-1$ & $8.79-1$ & $.55-1$ & $.29-1$ & $8.04-1$ & $7.86-1$ & $81-1$ & $.84-1$ & $94-1$ & $8.08-1$ \\
\hline $4 \mathrm{~s}$ & $2.66-1$ & $2.58-1$ & $2.47-1$ & $2.36-1$ & $2.27-1$ & $2.20-1$ & $2.18-1$ & $2.18-1$ & $2.20-1$ & $2.24-1$ & $2.28-1$ \\
\hline $4 p$ & $1.58-1$ & $1.42-1$ & $1.23-1$ & $1.05-1$ & $8.92-2$ & $7.84-2$ & $7.32-2$ & $7.45-2$ & $8.30-2$ & $9.95-2$ & $1.25-1$ \\
\hline $4 d$ & $1.29-1$ & $1.15-1$ & $1.03-1$ & $9.14-2$ & $8.21-2$ & $7.57-2$ & $7.25-2$ & $7.25-2$ & $7.54-2$ & $.06-2$ & $8.74-2$ \\
\hline $4 \mathrm{f}$ & $1.73-1$ & $1.74-1$ & $1.73-1$ & $1.69-1$ & $1.66-1$ & $1.63-1$ & $1.62-1$ & $1.63-1$ & $1.65-1$ & $1.66-1$ & $1.68-1$ \\
\hline \multicolumn{12}{|l|}{$3 p-$} \\
\hline $3 \mathrm{~d}$ & -2 & $15+1$ & 1.16 & $18+1$ & $22+1$ & $1.27+1$ & $1.34+1$ & $42+1$ & $1.52+1$ & 1.6 & $1.77+1$ \\
\hline $4 \mathrm{~s}$ & & 1 & $4.4 \varnothing$ & -1 & & $2.54-1$ & 2.3 & & & & $3.73-1$ \\
\hline $4 p$ & $1.34+0$ & $1.27+0$ & $1.19+0$ & $1.11+0$ & $1.04+0$ & $9.86-1$ & 9.58 & $9.49-1$ & 9.5 & 9.6 & $9.87-1$ \\
\hline $4 \mathrm{~d}$ & $7.03-1$ & $6.11-1$ & $5.35-1$ & $4.71-1$ & $4.22-1$ & $3.91-1$ & $3.82-1$ & $4.00-1$ & $4.48-1$ & $5.29-1$ & $6.47-1$ \\
\hline $4 \mathrm{f}$ & $8.48-1$ & $8.67-1$ & $8.76-1$ & $8.76-1$ & $8.79-1$ & $8.97-1$ & $9.33-1$ & $9.87-1$ & $1.06+0$ & $1.13+0$ & $1.21+0$ \\
\hline \multicolumn{12}{|c|}{ 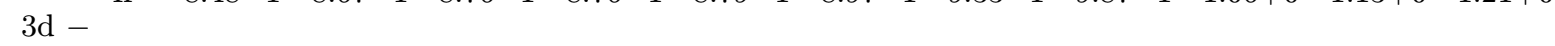 } \\
\hline $4 \mathrm{~s}$ & $1.40+0$ & $\vdash 0$ & -1 & $.53-1$ & -1 & $4.40-1$ & $3.44-1$ & $2.82-1$ & $2.43-1$ & $.20-1$ & $2.07-1$ \\
\hline $4 p$ & & & & & & 1.20 & & & -1 & -1 & +0 \\
\hline $4 \mathrm{~d}$ & $6+0$ & 3.5 & 3.1 & 2.8 & 2.6 & $2.38+0$ & 2.2 & 2.1 & +0 & +0 & $2.07+0$ \\
\hline $4 \mathrm{f}$ & $6.30+0$ & $6.82+0$ & $7.34+0$ & $7.46+0$ & $7.35+0$ & $7.38+0$ & $7.71+0$ & $8.40+0$ & $9.43+0$ & $1.08+1$ & $1.25+1$ \\
\hline \multicolumn{12}{|c|}{ 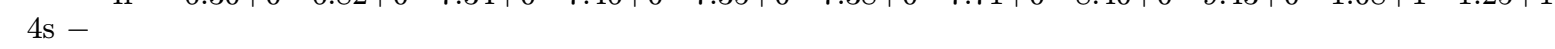 } \\
\hline $4 p$ & $2.56+1$ & $2.58+1$ & 2.67 & $2.87+1$ & $3.03+1$ & $3.08+1$ & $3.15+1$ & $3.31+1$ & & +1 & $4.21+1$ \\
\hline $4 \mathrm{~d}$ & $3.47+0$ & $3.71+0$ & $4.14+0$ & $4.31+0$ & $4.08+0$ & $3.73+0$ & $3.51+0$ & $3.43+0$ & $3.42+0$ & $3.44+0$ & $3.44+0$ \\
\hline $4 \mathrm{f}$ & $1.02+0$ & $1.08+0$ & $1.15+0$ & $1.13+0$ & $1.01+0$ & $8.83-1$ & $7.88-1$ & $7.32-1$ & $7.00-1$ & $6.82-1$ & $6.73-1$ \\
\hline \multicolumn{12}{|c|}{ 然 } \\
\hline $4 d$ & 4. & 5. & 6. & . & & 5.9 & & & -1 & +1 & $7+1$ \\
\hline \multicolumn{12}{|c|}{ 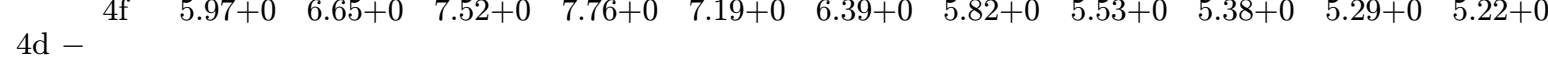 } \\
\hline $4 \mathrm{f}$ & $7.31+1$ & $8.73+1$ & $1.07+2$ & $1.16+2$ & $1.11+2$ & $1.00+2$ & $9.44+1$ & $9.45+1$ & $9.85+1$ & $1.04+2$ & $1.11+2$ \\
\hline
\end{tabular}




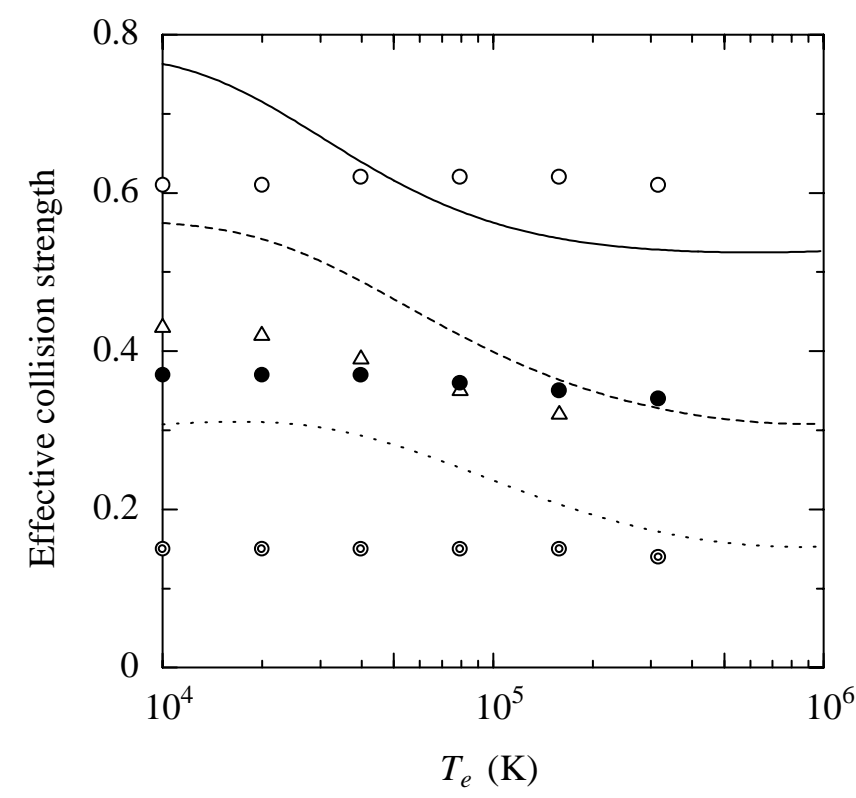

Fig. 1. Effective collision strengths of the $3 \mathrm{~s}-4 \mathrm{p}$ excitation for Al III, Si IV and S VI as a function of temperature (in K). Al III: - present, $\bigcirc$ Dufton \& Kingston (1987), $\triangle$ Mitroy \& Norcross (1989), Si IV: - - - present, • Dufton \& Kingston (1987), S VI: - - - present, ๑ Dufton \& Kingston (1987)

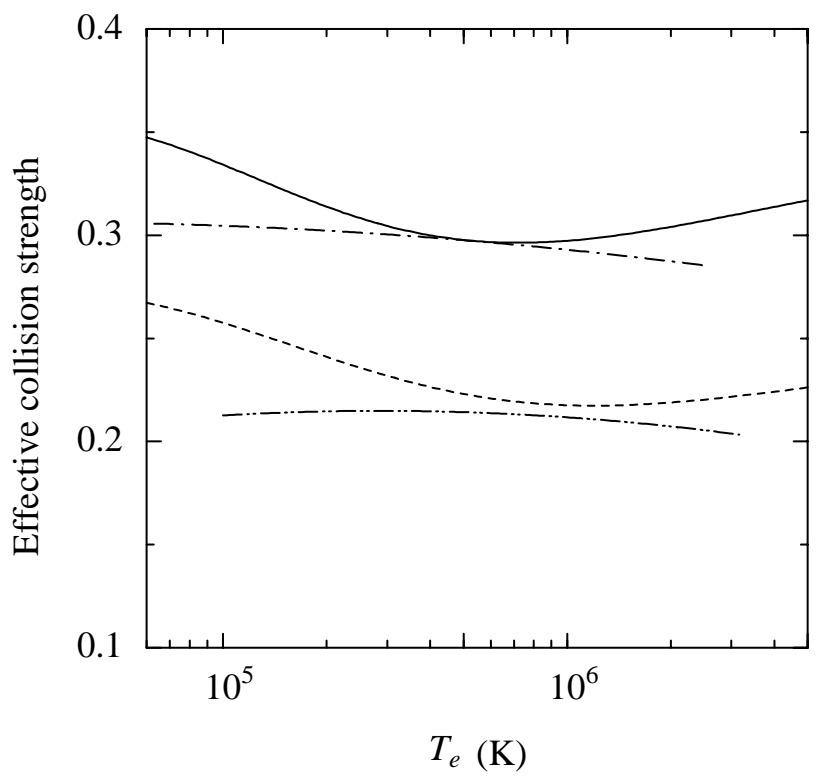

Fig. 2. Effective collision strengths of the $3 \mathrm{~s}-4 \mathrm{~s}$ excitation for Ar VIII and Ca X as a function of temperature (in K). Ar VIII: — present, - - - Keenan et al. (1996), Ca X: - - - present, - . - Keenan et al. (1996)

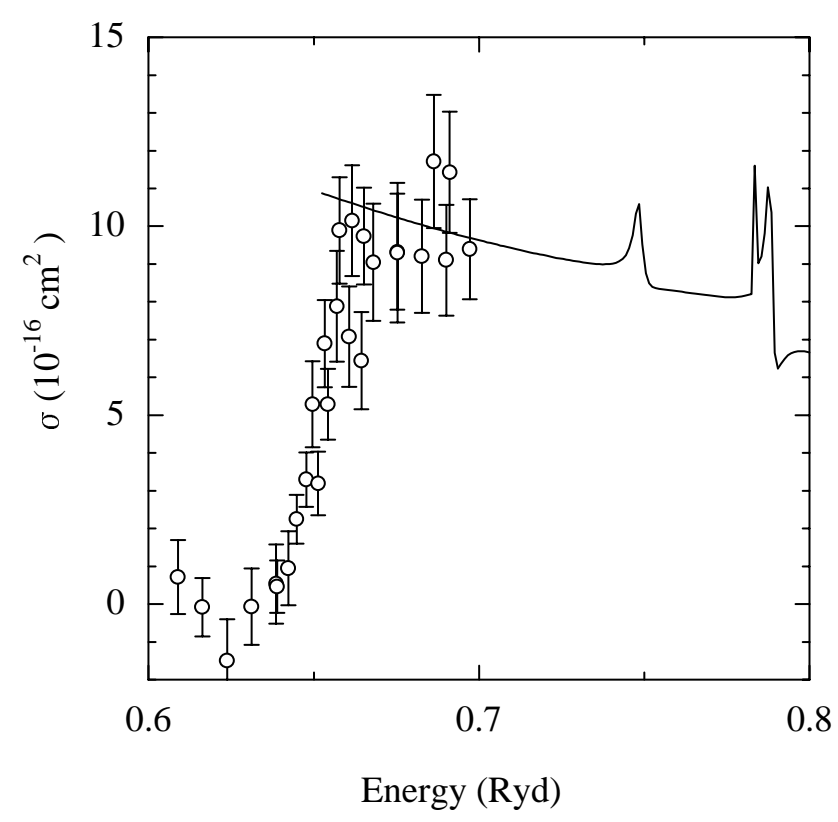

Fig. 3. Cross section for the $3 s-3 p$ excitation of $\mathrm{Si} I V$ as a function of electron energy (in Ryd). The excitation energy of this transition is 0.6524 Ryd. — present, $\bigcirc$ the experiment of Wåhlin et al. (1991)

section data. In this sense, the agreement is quite good. For Ar VIII, a similar agreement is obtained between the present calculation and the experiment (see Paper I, where a comparison of the cross section for Ar VIII with several other calculations has also been made). Since no experimental data are available to test the resonance structure in the cross sections for the present ions, it is difficult to critically assess the accuracy of the present rate coefficients. From other R-matrix calculations for other ions, however, the values given in Tables $3-7$ should be reliable for practical applications.

Acknowledgements. The R-matrix code used here has been kindly provided to the present authors by Dr. K.A. Berrington under the UK-Japan collaboration program on the theory of atomic collision. The present work was supported by a Grantin-Aid for Scientific Research on Priority Area "Atomic Physics of Multicharged Ions" from the Ministry of Education, Science and Culture of Japan.

\section{References}

Arnaud M., Rothenflug R., 1985, A\&AS 60, 425

Berrington K.A., Burke P.G., Dourneuf M. Le, et al., 1978, Comput. Phys. Commun. 14, 367

Berrington K.A., Burke P.G., Butler K., et al., 1987, J. Phys. B 20, 6379

Burke P.G., Robb W.D., 1975, Adv. At. Mol. Phys. 11, 143

Clementi E., Roetti C., 1974, Atomic Data Nucl. Data Tab. 14, 177

Dufton P.L., Kingston A.E., 1987, J. Phys. B 20, 3899 
Flower D.R., Nussbaumer H., 1975, A\&A 42, 265

Guo X.Q., Bell E.W., Thompson J.S., et al., 1993, Phys. Rev. A $47, \mathrm{R} 9$

Henry R.J.W., Rountree S.P., Smith Ed R., 1981, Comput. Phys. Commun. 23, 233

Hibbert A., 1975, Comput. Phys. Commun. 9, 141

Keenan F.P., 1988, Sol. Phys. 116, 279

Keenan F.P., 1993, in: Silver and Kahn (eds.) UV and X-ray Spectroscopy of Laboratory and Astrophysical Plasmas. Cambridge Univ. Press, p. 44

Keenan F.P., Dufton P.L., Kingston A.E., 1986, A\&A 169, 319

Keenan F.P., Roche I.J., Foster V.J., Mohan M., 1996, Phys. Scr. 54, 163
Mitroy J., Norcross D.W., 1989, Phys. Rev. A 39, 537

Moore C.E., 1971, Atomic Energy Levels, Natl. Stand. Ref. Data Ser. 35 Natl. Bur. Stand., Washington DC, Vol. I

Nakazaki S., Okahara M., Itikawa Y., 1996, J. Phys. Soc. Jpn. 65, 2472 (Paper I)

Takagishi K., Ohkura M., Nakazaki S., 1995, Comput. Phys. Commun. 85, 293

Wåhlin E.K., Thompson J.S., Dunn G.H., et al., 1991, Phys. Rev. Lett. 66, 157

Wiese W.L., Smith M.W., Miles B.M., 1969, Atomic Transition Probabilities, Natl. Bur. Stand. (U.S.), Natl. Stand. Ref. Data Ser. 22 U.S. GPO, Washington DC, Vol. 2 